\title{
Femtosecond pulses from a continuously self-starting passively mode-locked Ti:sapphire laser
}

\author{
U. Keller, G. W. 'tHooft,' W. H. Knox, and J. E. Cunningham \\ ATET Bell Laboratories, Crawfords Corner Road, Holmdel, New Jersey 07733
}

Received February 17, 1991

\begin{abstract}
We show that an external coupled cavity containing a nonlinear quantum-well reflector can continuously selfstart a dispersion-compensated Ti:sapphire laser, which produces stable time-transform-limited pulses as short as $70 \mathrm{fs}$ in a $\mathrm{TEM}_{00}$ mode. In this mode of operation, the quantum wells do not control the mode-locking process, as in previous research on the resonant passive mode-locked laser. By separating the mode-locking and starting processes, we show that the presence of higher-order spatial modes is not required to start or sustain mode locking.
\end{abstract}

Recently much emphasis has been placed on different mode-locking techniques for the Ti:sapphire laser with the aim of achieving broadly tunable short pulses with relatively high average power for spectroscopic applications. These techniques include acousto-optic mode locking, ${ }^{1}$ additive-pulse mode locking, ${ }^{2}$ resonant passive mode locking ${ }^{3}$ (RPM), moving-mirror mode locking, ${ }^{4}$ and intracavity passive mode locking with a dye jet ${ }^{5}$ and a colored glass filter. ${ }^{6}$ More recently a remarkable and interesting new self-mode-locking effect for the Ti:sapphire laser was introduced by Spence et $a l .{ }^{7}$ that generated pulses as short as $60 \mathrm{fs}$ with a dispersioncompensated Ti:sapphire laser. In the presence of higher-order spatial modes and critical misalignment of the cavity, the laser intermittently self-mode locks and then falls back into $\mathrm{cw}$ operation. It has been suggested that higher spatial modes are required in order to start and sustain mode locking ${ }^{7}$ and that a self-focusing effect can decrease the resonator losses and compensate for the linear misalignment of the cavity. ${ }^{8}$ In this Letter we show that a nonlinear reflection from a coupled cavity can in a controlled manner continuously self-start this mode-locking process and obtain pulses as short as $70 \mathrm{fs}$ in a $\mathrm{TEM}_{00}$ mode. The nonlinear reflection arises from a quantum-well reflector in a weakly coupled cavity similar to that previously used for the RPM laser. ${ }^{3}$ In the RPM case, the nonlinear cavity is more strongly coupled and the quantum-well nonlinearity governs the mode locking. Although the resonant quantum-well nonlinearity limits the selfstarting tuning range, it separates the starting mechanism from the self-mode-locking mechanism and helps in the understanding of the latter process. Our experimental observations support the idea of an ultrafast self-focusing effect in the Ti:sapphire rod as the responsible mode-locking mechanism even without any higher spatial modes.

We use a modified Spectra-Physics Model $3900 \mathrm{Ti}$ :sapphire laser with a single birefringent tuning plate. We asymmetrically extended the high-reflector side of the cavity by adding two SF10 prisms for dispersion compensation. ${ }^{9}$ The axial mode-beating frequency (i.e., the pulse repetition rate) is $\approx 133.6 \mathrm{MHz}$. We measured a slope efficiency of $20 \%$ and a pump threshold of $1.34 \mathrm{~W}$ at a wavelength of $854 \mathrm{~nm}$ using all lines of the argon-ion pump laser. The nonlinear coupled cavity is identical to that of the RPM laser, ${ }^{3}$ with the exception that a variable overall output coupler is formed by a halfwave plate and a polarizing beam splitter, and a variable aperture inside the Ti:sapphire laser was used to achieve femtosecond pulses. The purpose of this aperture will be discussed further.

We simultaneously monitor the laser output with a rf spectrum analyzer, a real-time noncollinear autocorrelator, a fast photodiode and a sampling scope, and an optical spectrum analyzer. We force the laser into $\mathrm{TEM}_{00}$ mode operation and start the mode locking with the quantum-well reflector. Without the nonlinear coupled cavity the laser does not start to mode lock. To achieve femtosecond mode-locked pulses we have to decrease an aperture inside the Ti:sapphire cavity (Fig. 1), which at a pump power of $5.4 \mathrm{~W}$ reduces the average output power from $800 \mathrm{~mW}$ (open aperture) to $\leq 400 \mathrm{~mW}$. At higher pump power we have to decrease the aperture even more. To prevent multiple pulsing we have to reduce the aperture to an average output power of $\leq 240 \mathrm{~mW}$. Under this condition we observe stable transform-limited pulses as short as $73 \mathrm{fs}$ assuming a hyperbolic-secant pulse shape [Fig. 2(a)] with an optical spectral width of $11.1 \mathrm{~nm}$ [Fig. 2(b)] at a center wavelength of $852 \mathrm{~nm}$. The resulting timebandwidth product is 0.33 , which is transform limited within experimental errors. We measured the amplitude noise spectrum $(>200 \mathrm{~Hz})$ of this laser, and, as in case of colliding-pulse mode-locked (CPM) lasers ${ }^{10}$ the amplitude noise is dominated by the argon-ion pump laser. The long-term stability is excellent because the laser is started in a welldefined manner with no uncontrolled breakdown into $\mathrm{cw}$ operation.

When the laser is mode locked, we can block the quantum-well reflector in the coupled cavity and ob- 


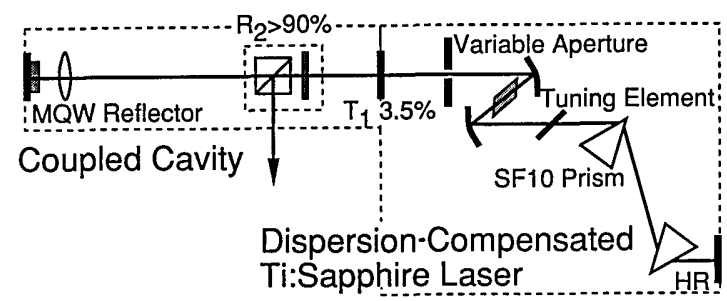

Fig. 1. Cavity setup. MQW, multiple quantum well.
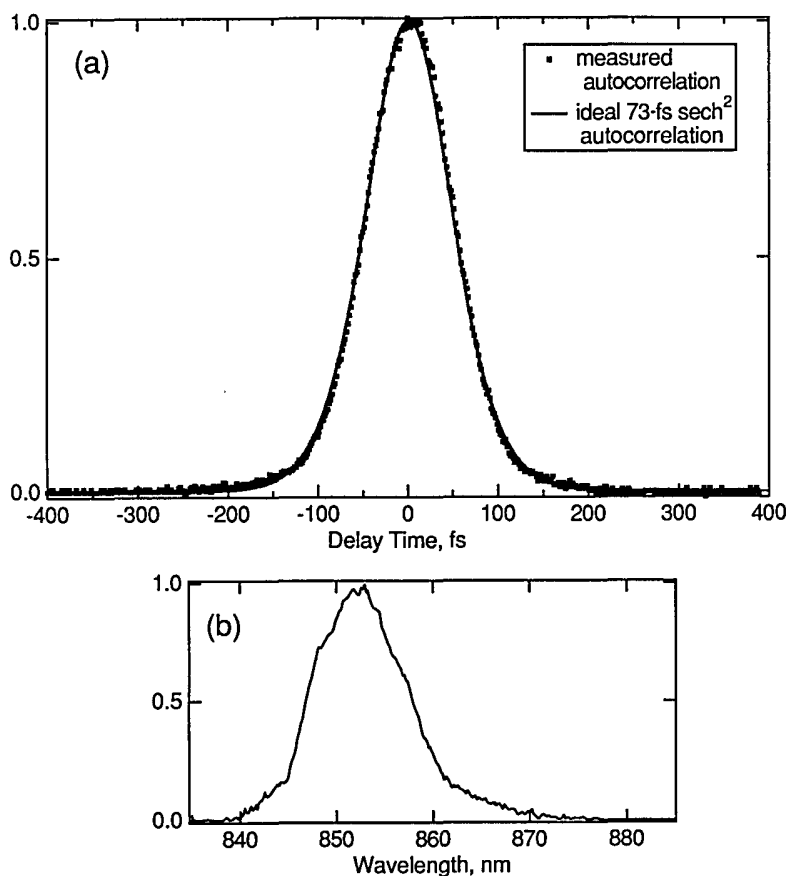

Fig. 2. (a) Autocorrelation for 73-fs pulse ( $\operatorname{sech}^{2}$ pulse shape), (b) optical spectrum with $11.1 \mathrm{~nm}$ FWHM.

serve that the laser maintains mode locking for a period from minutes to as long as hours depending on outside physical perturbations. While keeping the external cavity blocked, we can stop the modelocked operation by briefly interupting the beam in the main cavity. When we unblock the quantumwell reflector in the coupled cavity, the mode locking immediately starts again. In contrast, in case of the RPM Ti:sapphire ${ }^{3}$ or Nd:YLF (Ref. 11) laser the mode-locked operation ceases as soon as the coupled cavity is blocked. When we increase the coupling between the two cavities by reducing $R_{2}$ in Fig. 1, we observe strong instabilities because the quantumwell mode locking interferes with the self-modelocking process. When we detune the external cavity length and open up the aperture inside the Ti:sapphire laser, we move into the RPM regime $\left(R_{2}\right.$ is typically $30 \%$ to $50 \%$ ), and we observe stable picosecond pulse durations because the detuned coupled-cavity length produces the dominant bandwidth limitation. ${ }^{12}$

The measured spatial mode (using a chargepriming-device television camera with a frame grabber) is a TEM To $_{00}$ mode, which shows less than $8 \%$ noise variations from an ideal Gaussian mode, and the difference between $\mathrm{cw}$ and mode-locked operation is less than $6 \%$. The nonlinear coupled cavity has no influence on the spatial mode profile.
We measured the pulse width and the optical bandwidth as a function of the intracavity dispersion by the SF10 prism translation at matched cavity configuration. The laser beam is close to the apex of the first fixed prism, and the apex-to-apex distance between the prisms is $41.2 \mathrm{~cm}$. The measured pulse width shows little dependence on the intracavity dispersion. For comparison, Fig. 3(b) shows the pulse width of an infrared CPM laser ${ }^{13}$ for which identical prisms for dispersion compensation were used. Clearly, the dispersion in the Ti:sapphire laser is much less critical than in a CPM laser, and such a large difference cannot be explained only by the difference in photon cavity lifetimes.

The tuning range is shown in Fig. 4 superimposed on the quantum-well reflectivity curve. The $\mathrm{cw}$ Ti:sapphire laser tuning range is limited by mirror reflectivities and the birefringent plate. The tuning range over which the laser is continuously selfstarting is approximately $30 \mathrm{~nm}$ and is limited by the resonant nonlinearity in the external cavity as in case of the RPM Ti:sapphire laser. ${ }^{3}$ By simply turning the birefringent tuning element, the $73-\mathrm{fs}-$ long pulses at $852 \mathrm{~nm}$ broaden at a shorter wavelength to slightly chirped 110 -fs-long pulses at
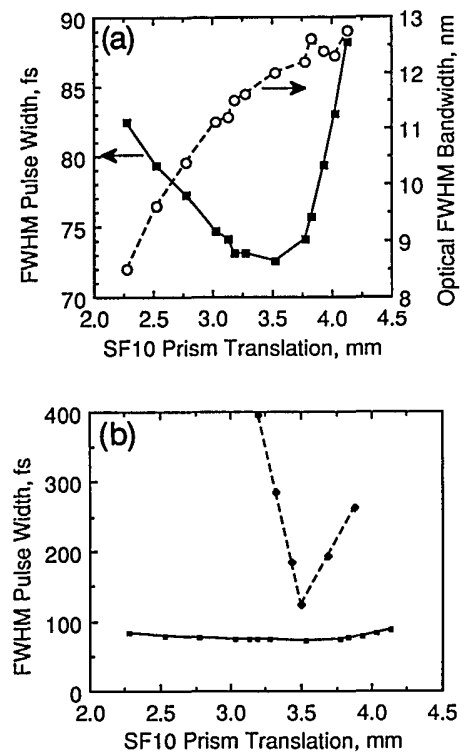

Fig. 3. (a) Scaling of pulse width (solid curve) and optical bandwidth (dashed curve) with relative intracavity dispersion (at zero translation of the prism the beam goes through the apex). (b) Pulse-width dependence for our Ti:sapphire laser (solid curve) and an infrared CPM laser (dashed curve, from Ref. 12).

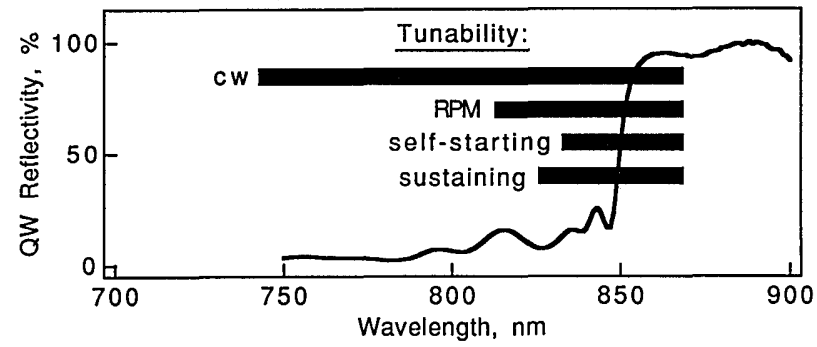

Fig. 4. Wavelength tuning range and quantum-well (QW) reflectivity. 
$835 \mathrm{~nm}$. The picosecond RPM tuning range ${ }^{3}$ is larger because in that case the two cavities were more strongly coupled $\left(R_{2}=10 \%\right)$. In addition, as discussed above when we block the quantum-well reflector, the laser sustains mode locking and can be tuned beyond the self-starting range. Because of the increasing loss modulation of the birefringent tuning element we could, however, only tune it to $\approx 828 \mathrm{~nm}$ at which the pulse width increased to $130 \mathrm{fs}$.

Our results support the suggestion ${ }^{8}$ that the mode-locking mechanism is a self-focusing effect inside the Ti:sapphire rod. When the cavity alignment is optimized for a stable TEM $_{00}$ mode at cw operation we need a limiting aperture inside the Ti:sapphire laser to generate femtosecond pulses. Under this condition, the average output power increases by $\approx 10 \%$ from the cw power level when the laser is mode locked. At constant pump power, to prevent any additional effects due to thermal lensing, the relative power increase becomes larger for smaller aperture sizes. We believe that the limiting aperture introduces a gain discrimination for $\mathrm{cw}$ (i.e., low-peak-power) and mode-locked (i.e., highpeak-power) operation. At high peak power a selffocusing effect inside the Ti:sapphire rod modifies the cavity mode in such a way that the beam waist at the aperture becomes smaller, which lowers losses in favor of a mode-locked operation. An instantaneous (Kerr-type) nonlinearity would produce a loss at the leading and at the trailing edges of the pulse that is sufficient to produce mode locking. The modelocking process has to be forced to start. In our case, an ultrafast reflectivity modulation from the quantum-well reflector is used. In the case of Spence et $a l .{ }^{7}$ the higher-order mode beating enhances the self-starting, and in the case of the intracavity passive mode locking ${ }^{5,6}$ the dye jet and the colored glass filter start the self-mode-locking process. We are able to reproduce the results of Spence et $a l .{ }^{7}$ without a limiting aperture but by misalignment of the cavity, which introduced higher spatial modes for $\mathrm{cw}$ operation. When, however, the laser mode locks (induced by the nonlinear coupled cavity) the mode is much closer to a TEM $_{00}$ mode, which again has higher gain. In addition, the weak dependence of the pulse width on the intracavity dispersion changes (Fig. 3) may be due to the fact that the laser needs the high peak power for the mode-locking process and only adjusts its optical bandwidth to compensate for the dispersion changes introduced by the prism translations.

In conclusion, we have shown that the quantumwell nonlinear coupled cavity, which mode locks a Ti:sapphire laser (RPM), ${ }^{3}$ can continuously self-start a different kind of mode locking. In contrast to previous research, ${ }^{7}$ we find that higher spatial modes are not required to initiate or sustain this modelocking process. Our experimental results support the idea that the mode-locking mechanism is an ultrafast self-focusing effect inside the Ti:sapphire laser rod. The tuning range of the self-starting is limited by the resonant quantum-well reflector to $\approx 30 \mathrm{~nm}$ but could be extended by, for example, a quantum-well reflector with a continuously varying band gap. We pay a power penalty by introducing a limiting aperture inside the Ti:sapphire laser cavity, but with an intensity-dependent laser cavity design it should be possible to get the full output power.

The authors thank T. Sizer II and D. W. Taylor for their help with the frame grabber.

*Permanent address, Philips Research Laboratories, Prof. Holstaan, 5600 JA Eindhoven, The Netherlands.

\section{References}

1. J. D. Kafka, A. J. Alfrey, and T. Baer, in Ultrafast Phenomena VI, T. Yajima, K. Yoshihara, C. B. Harris, and S. Shionoya, eds. (Springer-Verlag, Berlin, 1988), p. 64 .

2. J. Goodberlet, J. Wang, J. G. Fujimoto, and P. A. Schulz, Opt. Lett. 14, 1125 (1989).

3. U. Keller, W. H. Knox, and H. Roskos, Opt. Lett. 15, 1377 (1990).

4. P. M. W. French, S. M. J. Kelly, and J. R. Taylor, Opt. Lett. 15, 378 (1990).

5. Y. Ishida, N. Sarukura, and H. Nakano, in Digest of Ultrafast Phenomena VII (Springer-Verlag, Berlin, 1990), p. 75.

6. N. Sarukura, Y. Ishida, T. Yanagawa, and H. Nakano, Appl. Phys. Lett. 57, 229 (1990).

7. D. E. Spence, P. N. Kean, and W. Sibbett, Opt. Lett. 16, 42 (1991).

8. M. Piché, N. McCarthy, and F. Salin, in Digest of Annual Meeting of the Optical Society of America (Optical Society of America, Washington, D.C., 1990), paper MB8.

9. R. L. Fork, O. E. Martinez, and J. P. Gorden, Opt. Lett. 9, 150 (1984).

10. G. W. 'tHooft, U. Keller, W. H. Knox, and J. E. Cunningham, in Digest of Conference on Lasers and Electro-Optics (Optical Society of America, Washington, D.C., 1991), paper JMA6.

11. U. Keller, T. K. Woodward, D. L. Sivco, and A. Y. Cho, Opt. Lett. 16, 390 (1991).

12. H. A. Haus, U. Keller, and W. H. Knox, J. Opt. Soc. Am. B 8, 1252 (1991).

13. W. H. Knox and F. A. Beisser, in Digest of Ultrafast Phenomena VII (Springer-Verlag, Berlin, 1990), p. 20, Fig. 2a. 